\title{
Aortic Intima-Media Thickness and Mean Platelet Volume in Children With Type 1 Diabetes Mellitus
}

\author{
Murat Ersoy ${ }^{1}$; Hatice Nilgun Selcuk Duru ${ }^{2, ~}{ }^{\text {; }}$ Murat Elevli ${ }^{2}$; Ozlem Ersoy ${ }^{2}$; Mahmut \\ Civilibal $^{2}$ \\ ${ }^{1}$ Department of Pediatrics, Istanbul Education and Research Hospital, Istanbul, Turkey \\ ${ }^{2}$ Department of Pediatrics, Haseki Education and Research Hospital, Istanbul, Turkey \\ ${ }^{*}$ Corresponding author: Hatice Nilgun Selcuk Duru, Department of Pediatrics, Haseki Education and Research Hospital, Istanbul, Turkey. Tel: +90-2125294400, E-mail: nilgunduru@ \\ yahoo.com
}

Received: February 5, 2015; Revised: February 28, 2015; Accepted: March 10, 2015

\begin{abstract}
Background: Diabetes mellitus type 1 is the most common endocrine metabolic disorder occurring in childhood and adolescence due to the autoimmune destruction of pancreatic beta cells as a result of various environmental factors interacting with an underlying genetic predisposition. Diabetes is a risk factor for early onset atherosclerosis, and the high mortality rate seen in these patients is partially related to cardiovascular diseases.

Objectives: This study was conducted to compare mean platelet volume as a marker of early atherosclerosis with aortic intima-media thickness in children with type 1 diabetes and to identify its correlation with known cardiovascular risk factors.

Patients and Methods: The study included 27 patients between age range of 6 and 17 years that were diagnosed with type 1 diabetes and 30 healthy children of the same age range who did not have any chronic disease. In both groups, we used the color Doppler ultrasound to measure children's aortic intima-media thickness and identify their mean platelet volumes.

Results: There was no significant difference between the groups regarding gender distribution, age, High-Density Lipoprotein (HDL) and Low-Density Lipoprotein (LDL) cholesterol levels $(\mathrm{P}>0.05)$. Also no significant difference could be documented between the patient and control groups regarding the aortic intima-media thickness and mean platelet volume $(\mathrm{P}>0.05)$. However, there was a significant correlation between aortic intima-media thickness and mean platelet volume $(r=0.351 ; P<0.05)$.

Conclusions: In the present study, there was no evidence of early atherosclerosis in children with type 1 diabetes. However, mean platelet volume having a significant correlation with aortic intima-media thickness may be useful as an early marker of atherosclerosis.
\end{abstract}

Keywords: Atherosclerosis; Intima-Media Thickness; Children; Mean Platelet Volume; Type 1 Diabetes Mellitus

\section{Background}

Diabetes mellitus type 1 is the most common endocrine metabolic disorder occurring in childhood and adolescence due to the autoimmune destruction of pancreatic beta cells as a result of various environmental factors interacting with an underlying genetic predisposition (1). Diabetes is a risk factor for early onset atherosclerosis, and the high mortality rate seen in these patients is partially related to cardiovascular diseases.

In the pathogenesis of atherosclerosis, contacting with subendothelial tissue activates macrophages and platelets; thus, it has an active role in the inflammation process. Platelets have a key role in the development of acute complications of atherosclerosis. The first step in the development of atherosclerosis is the platelets' adhesion to the endothelium. Acute coronary syndromes are precipitated by a common pathophysiologic mechanism including rupture of atheromatous plaque, activation of coagulation cascade, and adhesion, activation and aggregation of platelets. During this cycle, larger platelets with higher volume get into circulation. As larger platelets are metabolically more active, the volume of the platelet is one of the determinants of platelet function. In addition to the studies reporting that increase in the mean platelet volume (MPV) is an early indicator of inflammatory process and increased platelet activation, there are publications claiming that it constitutes a new risk factor for atherosclerosis (2).

Initial manifestation of atherosclerosis is the lipid storage of systemic arteries on intima-media layers (3, 4). Measuring intima-media thickness and elasticity of blood vessels has been an excellent non-invasive method for detection of early atherosclerosis, and carotid artery, which can be non-invasively imaged and easily scanned, has been used for this measurement (5-9). Autopsy studies conducted on young bodies have shown that initial atherosclerotic changes occur in the abdominal aorta (10). Recent studies conducted following the latest developments in ultrasound techniques have remarked that

Copyright (C) 2015, Growth \& Development Research Center. This is an open-access article distributed under the terms of the Creative Commons Attribution-NonCommercial 4.0 International License (http://creativecommons.org/licenses/by-nc/4.0/) which permits copy and redistribute the material just in noncommercial usages, provided the original work is properly cited. 
it would be more significant to measure the thickness of aortic intima-media as an early indicator of atherosclerosis (11).

\section{Objectives}

In the present study, we aimed to detect early atherosclerosis by measuring aortic intima-media thickness in children and adolescents that had been followed up due to the diagnosis of type 1 diabetes, and to examine whether MPV could be a useful indicator in detection of atherosclerosis. Additionally, we analyzed the correlation of metabolic control and atherosclerosis with some parameters related to the risk factors. The study was approved by the Ethics Committee of the Hospital.

\section{Patients and Methods}

This prospective case-control study was conducted in the Child Health and Diseases Clinic of Haseki Education and Research Hospital, Istanbul. 27 patients that had been followed up in pediatric clinic and diagnosed with DM type 1 according to the ADA-2010 criteria were included in the study. The control group was composed of 30 children that applied to the pediatric clinic, but did not have any known chronic diseases.

The Ethics Committee of the Hospital approved the study. Both groups and their families were informed of the study. We obtained informed written consents from all families and those patients who were 12 years of age and above. Medical and laboratory practices within the scope of the study were applied according to the Ethical Principles for Medical Research Involving Human Subjects stated in the Declaration of Helsinki.

After obtaining detailed histories of all the children, they were physically examined and data was recorded on forms prepared for this study. Data recorded on the forms included age (year), sex, height $(\mathrm{cm})$, weight $(\mathrm{kg})$, body mass index (BMI) $\left(\mathrm{kg} / \mathrm{m}^{2}\right)$, consanguinity between the parents, presence of chronic diseases within the family, measurements of systolic and diastolic arterial blood pressure (mmHg), exposure to smoking, age at disease onset, age at diagnosis, doses of insulin, presenting symptoms and complaints, waist-to-hip ratio, and the laboratory findings including $\mathrm{Hb}(\mathrm{g} / \mathrm{dL}), \operatorname{Hct}(\%), \mathrm{MPV}(\mathrm{fL})$, total cholesterol (mg/dL), HDL-C (mg/dL), LDL-C (mg/dL), triglycerides $(\mathrm{mg} / \mathrm{dL}), \mathrm{HgA}_{1 \mathrm{C}}(\%)$, glucose $(\mathrm{mg} / \mathrm{dL})$, insulin (IU/mL), C-peptide (ng/mL) and microalbumin (mg/day).

\subsection{Laboratory Tests}

After a full night of fasting, blood samples were collected from both patients and control groups in the morning for the measurement of blood lipids, glucose, and insulin and also for other tests. Blood samples were centrifuged in gel tubes ( $2500 \mathrm{~g}$ for 5 minutes) and, after serum was effectively sealed off from the cell, serums were examined and analyzed in the central laboratory. Complete blood count was conducted using the fully automated Pentra
DX 120 blood cell counter. Total cholesterol, triglycerides, and HDL were determined with enzymatic colorimetric methods. Serum LDL levels were estimated using the Friedwald formula, TK $=\mathrm{LDL}+\mathrm{HDL}+\mathrm{TG} / 5$. While the photometric hexokinase method was applied for detecting glucose, the immunoturbidimetric method was used for measuring albumin levels in 24-hour urine. The Roche Diagnostics E170 device was used for the assessment of basal insulin levels. HbA1c levels were determined using the Microgel/ INTERLAB electrophoresis kit.

\subsection{Measurement of the Aortic Intima-Media Thickness}

Intima-media thickness measurements (AIMT) were carried out by the same radiologist in the Radiology Clinic of Haseki Education and Research Hospital using the Toshiba Aplio XR color Doppler ultrasound system in a quiet, dimly-lit environment where ambient temperature was between $20-22^{\circ} \mathrm{C}$. All cases were analyzed after at least 6 hours of fasting and under appropriate conditions where bradycardia or tachycardia did not develop. AIMT was measured at the optimal aorta diameter in the axial plane, and approximately at the $2.5 \mathrm{~cm}$ segment of the posterior wall in the sagittal plane using a $7.5 \mathrm{MHz}$ probe.

\subsection{Statistical Method}

Statistical analyses were conducted using the SPSS (Statistical Package for Social Sciences) Windows version 19.0. The average, standard deviation, ratio, and frequency values were used for the descriptive statistics of the data. Data distribution was tested using the Kolmogorov-Simirnov test. While parametric data was analyzed with $t$ test, proportional data was analyzed with chi-square test. Correlation between variables was examined using the Spearman-Pearson correlation. P $<0.05$ with $95 \%$ confidence interval was considered significant.

\section{Results}

27 patients diagnosed with type 1 diabetes that were followed up in Pediatric Clinic of the Ministry of Health Haseki Education and Research Hospital or applied to the general pediatric clinic, and also healthy children having no known chronic diseases participated in the study. Table 1 gives the summary of demographic characteristics and metabolic parameters of the study subjects. There was no statistical difference between the two groups regarding gender distribution. Glucose, $\mathrm{HgA}_{1 \mathrm{c}}$ and total cholestrol rates were statistically higher in the patient group. On the otherhand, C-peptide levels of the patient group were statistically lower than those of the control group. No significant difference could be observed between the HDL and LDL cholesterol levels of the two groups.

There was no statistically significant difference between the patient group and control group reagarding their MPV and AIMT (Table 1). However, there was a significant 
Ersoy Met al.

correlation between the MPV and AIMT ( $\mathrm{r}=0.351$; $\mathrm{P}=$ 0.036) of the patient group (Table 2). Similarly, MPV levels of the patients group were significantly correlated with total cholestrol level $(r=-0.426 ; P=0.013)$, whereas no significant correlation was found in control group (Table 3).

Table 1. Comparison of Demographic Characteristics, Metabolic Parameters, Mean Platelet Volume (MPV) and AOAIMT in Groups a, b

\begin{tabular}{lccc}
\hline Variables & Type 1 DM & Control & P Value \\
\hline Age, yr & $12.73 \pm 2.98$ & $11.46 \pm 2.71$ & 0.098 \\
Gender ratio, F/M & $13 / 14$ & $19 / 11$ & 0.249 \\
\hline BMI, kg/m 2 & $19.15 \pm 2.88$ & $17.7 \pm 3.71$ & 0.112 \\
\hline Glucose, mg/dL & $206.44 \pm 73.10$ & $88.80 \pm 7.33$ & 0.000 \\
\hline C-peptide, ng/mL & $0.63 \pm 0.50$ & $1.41 \pm 1.26$ & 0.004 \\
HbA $\mathbf{1 c}, \mathbf{m m o l} / \mathbf{m o L}$ & $0.43 \pm 3.09$ & $5.38 \pm 0.42$ & 0.000 \\
\hline T. cholesterol, mg/dL & $180.85 \pm 39.99$ & $156.23 \pm 23.92$ & 0.008 \\
HDL, mg/dL & $48.47 \pm 14.38$ & $50.77 \pm 11.65$ & 0.509 \\
\hline LDL, mg/dL & $100.19 \pm 24.21$ & $89.89 \pm 23.69$ & 0.111 \\
\hline MPV, fL & $8.74 \pm 0.96$ & $8.49 \pm 0.66$ & 0.264 \\
\hline AOAIMT, cm & $0.63 \pm 0.11$ & $0.58 \pm 0.12$ & 0.118 \\
\hline
\end{tabular}

a Abbreviations: BMI: body mass index, HDL: high-density lipoprotein , LDL: low-density lipoprotein , MPV: mean platelet volume.

$\mathrm{b}$ Data are presented as Mean \pm SD.

Table 2. Correlations of Mean Platelet Volume (MPV) and AOAIMT Values With Other Parameters of Study ${ }^{a}$

\begin{tabular}{lcccc}
\hline \multirow{2}{*}{ Study Group } & \multicolumn{2}{c}{ MPV } & \multicolumn{2}{c}{ AOAIMT } \\
\cline { 2 - 5 } & r & P Value & r & P Vale \\
\hline MPV, fL & 1.000 & & 0.351 & 0.036 \\
\hline AOAIMT, cm & & 0.351 & 0.036 & 1.000 \\
\hline Age, yr & 0.126 & 0.265 & 0.473 & 0.006 \\
\hline BMI, kg/m 2 & -0.133 & 0.255 & 0.467 & 0.007 \\
\hline Glucose, mg/dL & 0.138 & 0.246 & 0.121 & 0.274 \\
\hline T. cholesterol, mg/dL & -0.426 & 0.013 & -0.191 & 0.170 \\
\hline HDL, mg/dL & -0.314 & 0.055 & -0.148 & 0.231 \\
\hline LDL, mg/dL & -0.285 & 0.075 & 0.006 & 0.489 \\
\hline HbA, mmol/moL & -0.104 & 0.303 & -0.047 & 0.408 \\
\hline C-Peptide, ng/mL & 0.0034 & 0.432 & 0.201 & 0.157 \\
\hline Microalbuminuria, mg/d & 0.060 & 0.383 & 0.080 & 0.346 \\
\hline Gender & 0.038 & 0.425 & 0.079 & 0.348 \\
\hline
\end{tabular}

a Abbreviations: BMI: body mass index, HDL: high-density lipoprotein, LDL: low-density lipoprotein, MPV: mean platelet volume.
Table 3. Correlations of Mean Platelet Volume (MPV) and AOAIMT Values With Other Parameters of Control Group ${ }^{a}$

\begin{tabular}{lcccc}
\hline \multirow{2}{*}{ control Group } & \multicolumn{2}{c}{ MPV } & \multicolumn{2}{c}{ AOAIMT } \\
\cline { 2 - 5 } & r & Pvalue & r & P vale \\
\hline MPV, fL & 1.000 & & 0.209 & 0.134 \\
AOAIMT, cm & & 0.209 & 0.134 & 1.000 \\
Age, yr & 0.115 & 0.273 & 0.360 & 0.025 \\
BMI, kg/m & -0.031 & 0.436 & 0.345 & 0.031 \\
Glucose, mg/dL & 0.052 & 0.392 & 0.015 & 0.469 \\
T. cholesterol, mg/dL & 0.034 & 0.429 & 0.018 & 0.462 \\
\hline HDL, mg/dL & 0.276 & 0.070 & -0.167 & 0.189 \\
\hline LDL, mg/dL & 0.032 & 0.433 & 0.070 & 0.356 \\
\hline HbA $\mathbf{1 c}$, mmol/moL & 0.343 & 0.032 & 0.312 & 0.047 \\
\hline C-peptide, ng/mL & -0.142 & 0.228 & 0.168 & 0.187 \\
Microalbuminuria, mg/day & -0.202 & 0.142 & -0.013 & -0.066 \\
Gender & 0.168 & 0.187 & 0.474 & 0.364 \\
\hline
\end{tabular}

a Abbreviations: BMI: body mass index, HDL: high-density lipoprotein , LDL: low-density lipoprotein , MPV: Mean Platelet Volume.

\section{Discussion}

The incidence of diabetes type 1 is increasing almost in all countries and the annual increase is reported to be approximately $3 \%$ (1). The higher the prevalence of diabetes type 1, the higher become mortality and morbidity resulting from complications. Discovery of insulin has enabled prevention from acute complications of diabetes mellitus; however, chronic complications such as accelerated atherosclerosis, retinopathy, and nephropathy still constitute a problem for both physicians and patients.

Studies have indicated that conditions including endothelial dysfunction occurring in the early stages of atherosclerosis are triggered sooner in individuals diagnosed with a group of illnesses having enhanced proinflammatory load, such as diabetes, and that this process leads to development of cardiovascular diseases (CVDs) at an early age (12). Diabetes accelerates the natural course of atherosclerosis and causes, not only more disseminated atherosclerotic lesions, but also a greater number of coronary artery involvement. American Heart Association has established diabetes as a cardiovascular risk factor and it is currently regarded as a coronary artery disease equivalent (13).

Atherosclerotic heart disease is a preventable disease. Primary prevention can be achieved when disease-causing factors are identified in advance and changeable risk factors are eliminated. Therefore, early detection of the process using non-invasive easy methods is crucial in diseases leading up to atherosclerosis, such as diabetes.

Platelets are cell fragments that play an active role in the development of atherosclerosis and acute complications. Mean platelet volume (MPV) is a determinant of platelet functions and has a positive association with 
the indicators of platelet activity including aggregation, thromboxane A2, PF4, and $\beta$-thromboglobulin release (14). Larger platelets produce more prothrombotic factors and adhere more easily. Additionally, large platelets contain denser granules and secrete more serotonin and $\beta$-thromboglobulin in comparison to small platelets. Increased MPV is associated with greater in-vitro aggregation in response to ADP and collagen. Sharpe and Trinick (15) have compared MPV levels of patients having type $1 \mathrm{di}-$ abetes with MPV levels of a healthy control group and indicated that MPV level increases significantly in patients with diabetes as compared to the healthy control group, whereupon, they argued that especially large platelets are effective on microvascular and macrovascular complications of diabetes. Coban et al. (16) analyzed MPV value in adults and made a comparison between patients having impaired glucose tolerance with the patients diagnosed with diabetes. In the aforementioned study, they found the MPV levels to be high in both groups. Based on the assumption that morphology of platelets shows a change in diabetes, Brown et al. (17) measured the MPV levels and platelet numbers of patients having coronary artery disease without diabetes, of the patients with diabetes but without vascular complication, and of those diagnosed with both diabetes and vascular disease. After those measurements, they compared obtained results with the results of a healthy control group. That study which was conducted on adult patients indicated that although the number of platelets increased in all groups, there was a significant increase only in the group of diabetic patients with vascular diseases. It was also observed in the same study that there was a significant difference between the diabetic and non-diabetic groups with atherosclerosis; however, there was no significant difference in diabetic patients without vascular complications. These findings have given the impression that, contrary to the hypothesis claiming the size of a platelet is determined during platelet production and some elements like insulin generate new risk factors for atherosclerosis in diabetic patients by leading to production of larger platelets, presence of larger platelets may be a result or an indicator of atherosclerosis.

It is still controversial whether MPV anomalies are the reflection of a pre-existing abnormality in the main pathogenesis of the incidence or a secondary phenomenon resulting from atherosclerosis. In the present study, we could not find any significant difference between the MPV level of the patient $(8.74 \pm 0.96)$ and control (8.49 \pm 0.66 ) groups. This result suggested that the MPV level would increase in the more advanced stages of atherosclerosis. Our patients were in childhood age group and their duration of illness was short. Another possibility is that, as Brown et al. (17) stated in their study, the increase in the MPV level is not associated with diabetes; rather, it is a result of atherosclerosis occurring regardless of diabetes.

In our study, we also measured the intima-media thick- ness which is regarded as a marker of early atherosclerosis. We carried out measurements of aortic intima-media thickness (AIMT) which has been mostly supported by latest publications as a useful method for analyzing early atherosclerosis in young people compared to carotid artery. Jarvisalo et al. (9) examined the relation between AIMT and carotid artery intima-media thickness (CIMT) and found that both AIMT and CIMT were high in highrisk (diabetic and hypercholesterolemic) children; however, the increase in the AIMT was greater. Davis et al. (18) have concluded from the study in which they measured both aortic and carotid AIMT of 313 healthy men and 322 healthy women that abdominal aorta measurement is more applicable to young patients compared to the elderly, and less likely to miss data. Additionally, the same study indicated that increased age leads to a more significant increase in AIMT compared to CIMT. Harrington et al. (11) reported aortic intima-media thickness to be a more valuable indicator of early atherosclerosis in children diagnosed with diabetes mellitus type 1 as compared to carotid intima-media thickness. In that study, they found a significant correlation between aortic intima-media thickness and $\mathrm{HbA}_{1 \mathrm{C}}$ and lipids.

We have not found any signs of atherosclerosis in our patients with type 1 diabetes. These results maybe explain that atherosclerosis has not yet developed in our children and adolescent patients who had good metabolic management; moreover, this may be a result of regular and intensive insulin therapy.

Rabago Rodriguez et al. (19) have reported that intensive insulin therapy lowers the increase in carotid intimamedia thickness (CIMT). Although intensive insulin therapy is effective, we could not identify any statistical correlation between AIMT and insulin dose. At the same time, although elevation of systolic and diastolic blood pressure has a positive effect on the increase of CIMT (8, 20 ), the AIMT was not high in our study. This condition may have occurred due to the fact that patients included in the study were non-hypertensive. In the study, we identified a significant correlation between MPV, total cholesterol and AIMT values of the patient group. Pathansali et al. (21) have aimed to examine the effectiveness of primary hypercholesterolemia on MPV in the study they carried out on 8 patients without diabetes, coronary artery disease and stroke and 14 control subjects, and reported that isolated primary hypercholesterolemia has no significant effect on MPV unless it is not secondary to a chronic disease. It is, therefore, very important that, in our study, the positive correlation established between the MPV and cholesterol levels of the diabetic patient group could not be observed in the healthy control group. The other important result obtained in the study is the presence of a statistically significant correlation between the MPV and AIMT of diabetic patient group. One of the most remarkable findings of the study is the determination of the positive correlation between hypercholesterolemia which is a risk factor for atherosclerosis, AIMT as an early marker 
of atherosclerosis and MPV especially in the patients diagnosed with diabetes type 1 . Also, that finding should be discussed with the results of other studies.

In the light of these findings, the importance of analyzing children with diabetes type 1 for the presence of atherosclerosis, earlier diagnosis of the illness, taking required measures and preventing complications that may develop either in childhood or adulthood, should be emphasized here. Additionally, despite not obtaining any significant differences between AIMT and CIMT values of the patient and control groups, there was a correlation between AIMT and MPV in the patient group. The result may lead to more comprehensive studies for clarifying that correlation.

\section{References}

1. Onset features and subsequent clinical evolution of childhood diabetes over several years. Lipton RB, Drum ML, Danielson KK, Greeley SA, Bell GI, Hagopian WA. Pediatr Diabetes. 2011;12(4 Pt 1):326.

2. Mean platelet volume as an indicator of platelet activation: methodological issues. Park Y, Schoene N, Harris W. Platelets. 2002;13(5-6):301.

3. Associations of coronary heart disease risk factors with the intermediate lesion of atherosclerosis in youth. The Pathobiological Determinants of Atherosclerosis in Youth (PDAY) Research Group. McGill HJ, McMahan CA, Zieske AW, Sloop GD, Walcott JV, Troxclair DA, et al. Arterioscler Thromb Vasc Biol. 2000;20(8):1998.

4. Association between multiple cardiovascular risk factors and atherosclerosis in children and young adults. The Bogalusa Heart Study. Berenson GS, Srinivasan SR, Bao W, Newman W3, Tracy RE, Wattigney WA. N Engl J Med.1998;338(23):1650.

5. Ultrasonographically assessed carotid morphology and the risk of coronary heart disease. Salonen JT, Salonen R. Arterioscler Thromb. 1991;11(5):1245.

6. Endothelial dysfunction and increased arterial intima-media thickness in children with type 1 diabetes. Jarvisalo MJ, Raitakari M, Toikka JO, Putto-Laurila A, Rontu R, Laine S, et al. Circulation. 2004;109(14):1750.

7. Non-invasive detection of endothelial dysfunction in children and adults at risk of atherosclerosis. Celermajer DS, Sorensen KE, Gooch VM, Spiegelhalter DJ, Miller OI, Sullivan ID, et al. Lancet. 1992;340(8828):1111.
8. Carotid artery intima-media thickness in children with type $1 \mathrm{di}$ abetes. Jarvisalo MJ, Putto-Laurila A, Jartti L, Lehtimaki T, Solakivi T, Ronnemaa T, et al. Diabetes. 2002;51(2):493.

9. Increased aortic intima-media thickness: a marker of preclinical atherosclerosis in high-risk children. Jarvisalo MJ, Jartti L, NantoSalonen K, Irjala K, Ronnemaa T, Hartiala JJ, et al. Circulation. 2001;104(24):2943.

10. Effects of coronary heart disease risk factors on atherosclerosis of selected regions of the aorta and right coronary artery. PDAY Research Group. Pathobiological Determinants of Atherosclerosis in Youth. McGill HJ, McMahan CA, Herderick EE, Tracy RE, Malcom GT, Zieske AW, et al. Arterioscler Thromb Vasc Biol. 2000;20(3):836.

11. Aortic intima media thickness is an early marker of atherosclerosis in children with type 1 diabetes mellitus. Harrington J, Pena AS, Gent R, Hirte C, Couper J. J Pediatr. 2010;156(2):237.

12. Endothelial cell activation, injury, damage and dysfunction: separate entities or mutual terms? Blann AD. Blood Coagul Fibrinolysis. 2000;11(7):623.

13. Diabetes and atherosclerosis: epidemiology, pathophysiology, and management. Beckman JA, Creager MA, Libby P. JAMA. 2002;287(19):2570.

14. The relationship of the mean platelet volume and C-reactive protein levels with mortality in ischemic stroke patients. Arikanoglu A, Yucel Y, Acar A, Cevik MU, Akil E, Varol S. Eur Rev Med Pharmacol Sci. 2013;17(13):1774.

15. Mean platelet volume in diabetes mellitus. Sharpe PC, Trinick T. Q J Med. 1993;86(11):739.

16. The mean platelet volume in subjects with impaired fasting glucose. Coban E, Bostan F, Ozdogan M. Platelets. 2006;17(1):67. doi: 10.1080/09537100500220729. [PubMed:16308190]

17. Megakaryocyte ploidy and platelet changes in human diabetes and atherosclerosis. Brown AS, Hong Y, de Belder A, Beacon $\mathrm{H}$, Beeso J, Sherwood R, et al. Arterioscler Thromb Vasc Biol. 1997;17(4):802.

18. Measurement of aortic intimal-medial thickness in adolescents and young adults. Davis PH, Dawson JD, Blecha MB, Mastbergen RK, Sonka M. Ultrasound Med Biol. 2010;36(4):560.

19. Carotid intima-media thickness in pediatric type 1 diabetic patients. Rabago Rodriguez R, Gomez-Diaz RA, Tanus Haj J, Avelar Garnica FJ, Ramirez Soriano E, Nishimura Meguro E, et al. Diabetes Care. 2007;30(10):2599.

20. Carotid atherosclerosis in relation to systolic and diastolic blood pressure: Kuopio Ischaemic Heart Disease Risk Factor Study. Salonen R, Salonen JT. Ann Med. 1991;23(1):23.

21. Altered megakaryocyte-platelet haemostatic axis in hypercholesterolaemia. Pathansali R, Smith N, Bath P. Platelets. 2001;12(5):292. 